\title{
Effect of remote ischemic preconditioning on postoperative acute kidney injury among patients undergoing cardiac and vascular interventions: a meta-analysis
}

\author{
Bingjue $\mathrm{Li}^{1,2,3,4} \cdot$ Xiabing Lang ${ }^{1,2,3,4} \cdot$ Luxi Cao ${ }^{1,2,3,4} \cdot$ Yuchen Wang ${ }^{1,2,3,4}$. \\ Yingying Lu $\mathrm{Lu}^{1,2,3,4} \cdot$ Shi Feng ${ }^{1,2,3,4} \cdot$ Yi Yang ${ }^{1,2,3,4} \cdot$ Jianghua Chen $^{1,2,3,4}$. \\ Hong Jiang ${ }^{1,2,3,4}$
}

Received: 13 December 2015/Accepted: 25 March 2016/Published online: 18 April 2016

(C) The Author(s) 2016. This article is published with open access at Springerlink.com

\begin{abstract}
It is currently controversial whether remote ischemic preconditioning (RIPC) reduces the incidence of acute kidney injury (AKI) in patients undergoing cardiovascular interventions. The main objective of this metaanalysis was to investigate whether RIPC provides renal protection for patients undergoing cardiac or vascular surgery. We searched the PubMed database (1966-Oct 2015), Embase database (1966-Oct 2015), Google Scholar, Cochrane Library, ClinicalTrials Database and Open Grey. Then we conducted a meta-analysis of the randomized controlled trials that met the inclusion criteria of our study. The interventions included use of an inflatable tourniquet around the limbs or cross-clamping of the iliac arteries before surgery (RIPC groups) and general cardiovascular intervention (control groups). The main outcomes examined included the incidence of AKI; changes in acute kidney injury biomarkers; and use of renal replacement therapy. Other outcomes examined included in-hospital mortality and the lengths of hospital stay and intensive care unit (ICU) stay. Finally, we screened 26 eligible studies containing 6699 patients who underwent cardiac or
\end{abstract}

Hong Jiang

annie.jh@163.com

1 Kidney Disease Center, The First Affiliated Hospital, School of Medicine, Zhejiang University, Qinchun Road 79\#, Hangzhou 310003, People's Republic of China

2 Kidney Disease Immunology Laboratory, The Third Grade Laboratory, State Administration of Traditional Chinese Medicine of P.R. China, Hangzhou, People's Republic of China

3 Key Laboratory of Multiple Organ Transplantation, Ministry of Health, Hangzhou, People's Republic of China

4 Key Laboratory of Nephropathy, Zhejiang Province, Hangzhou, Zhejiang, People's Republic of China vascular interventions with RIPC $(\mathrm{n}=3343)$ or without RIPC $(\mathrm{n}=3356)$. The AKI incidence was decreased in the RIPC group as was the length of ICU stay. There were no differences in the changes in AKI biomarkers, use of renal replacement therapy or in-hospital mortality between the two groups. Remote ischemic preconditioning may decrease the occurrence of AKI in cardiovascular surgery patients. Since studies included have a significant heterogeneity, meta-analyses using a stricter inclusion criteria are needed to clarify the renoprotection effect of RIPC.

Keywords Remote ischemic preconditioning - Acute kidney injury - Cardiac and vascular interventions . Meta-analysis

\section{Introduction}

Acute kidney injury (AKI) is a serious post-operation complication in cardiac surgery patients [1]; its incidence ranges from 3 to $42 \%$ [2-10], and 1 to $5 \%$ of AKI patients require dialysis therapy $[1,2,4,9,11]$. The mortality of AKI patients has been reported to be as high as $40-80 \%$ [1, 7, 9]. Although this clinical problem is gaining increased attention, there are still no efficient methods to prevent AKI after cardiac and vascular interventions [5, 6, 12-14]. A double-blinded, placebo-controlled multicenter study conducted by Julier et al. [15] confirmed that sevoflurane preconditioning reduces the increase in postoperative plasma cystatin $\mathrm{C}$ (Cys $\mathrm{C}$ ) concentration; however, that trial did not investigate the relationship between sevoflurane preconditioning and perioperative AKI prevalence.

Remote ischemic preconditioning (RIPC) consists of cycles of transient non-fatal ischemia in one tissue to 
enhance the toleration of a subsequent prolonged fatal ischemia in distant organs [16]. The protective mechanism of RIPC to specific organs has been illustrated by several studies [14, 17, 18]; however, whether remote ischemic preconditioning has a positive clinical effect on renal function in cardiac and vascular surgery patients remains unclear. Some previous randomized controlled trials (RCTs) showed that RIPC reduces AKI incidence in cardiac surgery patients, but other studies had conflicting results. Several previous systematic reviews also demonstrated controversial results [19-23]. In recent years, more relevant RCTs have been carried out and published, so we performed a meta-analysis to verify the effect of RIPC on acute kidney injury in patients undergoing cardiovascular interventions.

\section{Methods}

\section{Study design}

Studies that met the following inclusion criteria were included in this meta-analysis: (1) RCT design; (2) study participants underwent some type of elective or acute cardiac or vascular surgery; (3) RIPC intervention, regardless of the duration or number of cycles; vessel occlusion models were also included; the control group intervention was standard treatment without RIPC or with sham RIPC; and (4) report of the incidence of AKI.

The primary outcome analyzed was the incidence of AKI. The secondary outcome measures included change in renal biomarkers after surgery, the use of renal replacement therapy, in-hospital mortality, the length of hospital stay and the length of intensive care unit (ICU) stay.

\section{Search strategy}

A literature search was conducted after establishing the inclusion criteria. We searched published articles in the PubMed (1966-Oct 2015), Embase (1966-Oct 2015), Google Scholar and Cochrane Library databases. We also searched ClinicalTrials.gov and Open Grey for unpublished and ongoing trials. There were no language or region restrictions. The following Medical Subject Heading terms and text words were used: ischemic preconditioning, cardiovascular surgical procedures, randomized controlled trial, controlled clinical trial, remote ischemic preconditioning. Titles and abstracts were screened by two authors ( $\mathrm{L}-\mathrm{xC}, \mathrm{Y}-\mathrm{cW})$ to guarantee their concordance with the inclusion criteria. Full text screening was conducted by the same two persons after preliminary screening if the article's eligibility could not be determined by screening the title and abstract.

\section{Data extraction}

Data extraction was performed by another two authors (Y$y L, S F)$ using a standardized data extraction form. Disagreements were resolved by a third person who served as an intermediary (B-jL) and made the final decision. Every trial was carefully assessed, and the following data were extracted: patient demographic characteristics, types of cardiovascular interventions, RIPC protocol, dose of contrast medium, AKI definition, incidence of AKI, in-hospital mortality, length of hospital stay, length of ICU stay, incidence of kidney replacement therapy, serum or plasma creatinine levels before and 24 and $48 \mathrm{~h}$ after surgery, and glomerular filtration rates (GFRs) at 24 and $48 \mathrm{~h}$ after surgery.
Fig. 1 Flowchart of article selection. $R C T$ randomized controlled trial

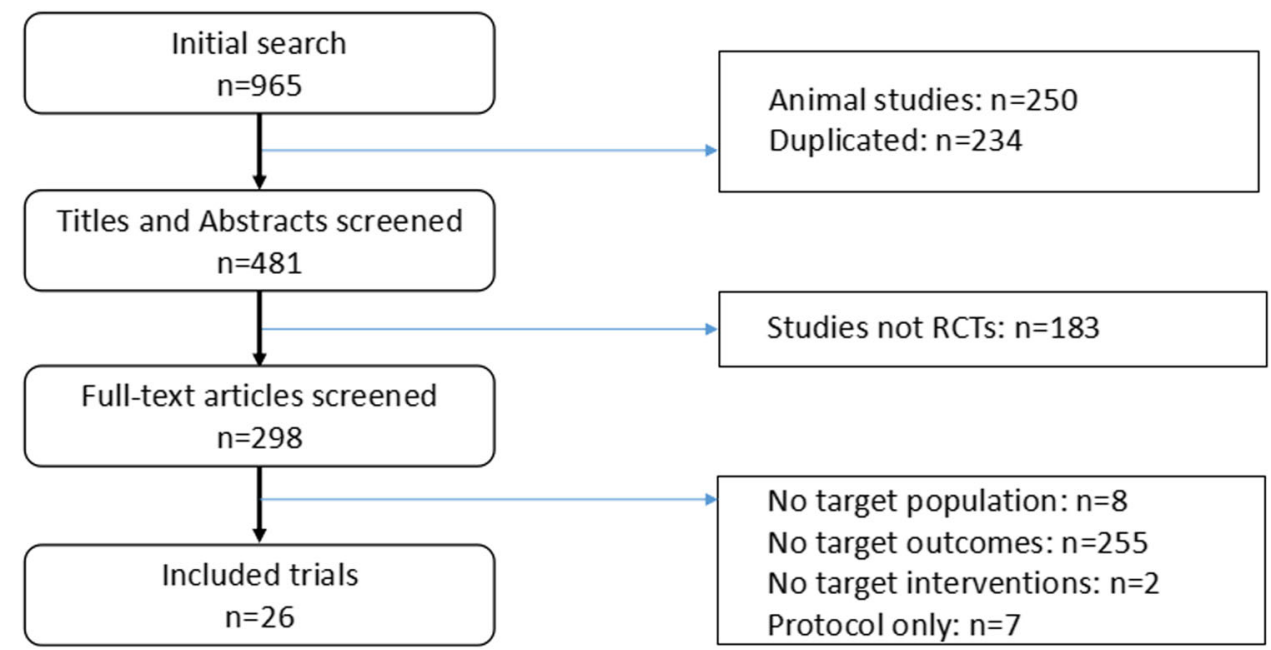




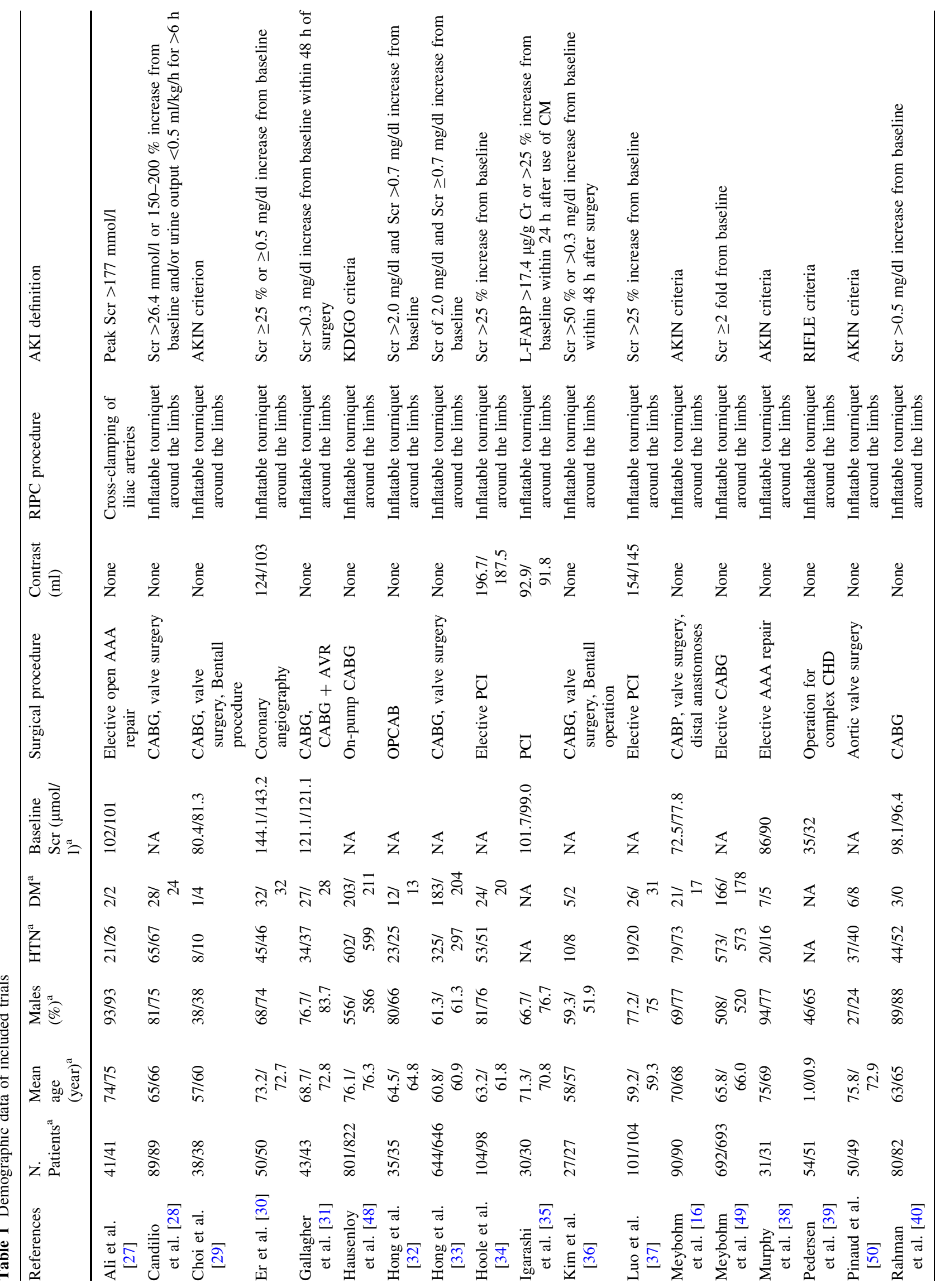




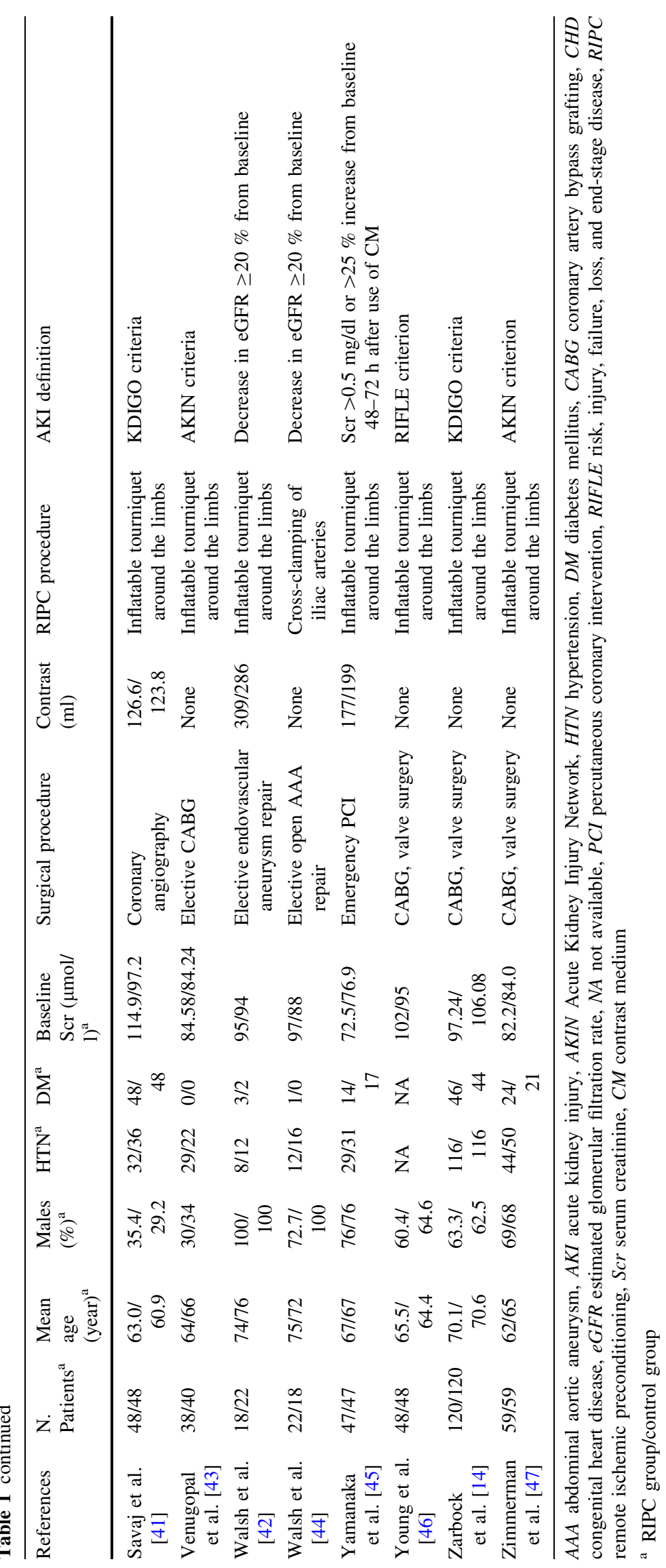




\section{Study validity assessment}

The Jadad scale was used to evaluate the methodological quality of the eligible trials. This scoring standard examines randomization, blinding, and explanation for withdrawals and dropouts [24, 25]. The modified Jadad scale, which includes the additional factor of item allocation concealment, was also used, with a score of 1-3 indicating low quality and a score of 4-7 indicating high quality [26]. Intention-to-treat (ITT) was also analyzed. We also judged risk of bias for each included study in terms of selection bias, performance bias, detection bias and attrition bias.

\section{Statistical analysis}

We mainly utilized the software Review Manager (RevMan) version 5.3 to analyze the extracted data (Copenhagen: The Nordic Cochrane Centre, The Cochrane Collaboration, 2014). Risk ratios (RRs) with $95 \%$ confidence intervals (CIs) were calculated for dichotomous outcomes, and mean differences with $95 \%$ CI were calculated for continuous outcomes. Statistical heterogeneity was assessed using the $\chi^{2}$ test, and we determined the percentage of total variation across studies using the Higgins $\mathrm{I}^{2}$ statistic. We compared our primary analysis with random-effects models using the Knapp-Hartung method to determine the robustness of the pooled effects.

\section{Results}

\section{Search results}

The search initially identified 965 articles; 234 duplicated articles and 250 animal studies were excluded. After title and abstract screening, 183 nonrandomized trials were excluded. Then, by full-text assessment of the remaining articles, we finally identified 26 eligible randomized controlled trials [14, 16, 27-50] (excluded articles: no target population: $\mathrm{n}=8$; no target outcomes: $\mathrm{n}=255$; no target interventions: $\mathrm{n}=2$; protocol only: $\mathrm{n}=7$, Fig. 1 ).

\section{Study characteristics}

A total of 6699 patients were enrolled in the 26 included studies, with 3343 patients randomized to the RIPC group and 3356 randomized to the control group. Sixteen studies examined cardiac surgery $[14,16,28,29,31-33,36,39$, $40,43,46-50]$, six studies examined percutaneous coronary intervention [30, 34, 35, 37, 41, 45], and four studies examined vascular surgery [27, 38, 42, 44]. The RIPC protocols were different between studies: 24 studies used an inflatable tourniquet around the limbs [14, 16, 27-50], and two studies used cross-clamping of the iliac arteries [27, 44]. The participants in one of the studies were children [39], while those of all other studies were adults. Seven studies applied contrast medium $[30,34,35,37,41$, $42,45]$. The key characteristics of the included studies are shown in Table 1.

\section{Quality assessment}

Two authors (YY, X-bL) independently assessed the quality of the 26 studies using the Jadad scale and the modified Jadad scale. Twenty-one $(80 \%)$ trials $[14,16$, $27-29,32-34,36-40,42-45,48-50]$ had a relatively high methodological quality based on the Jadad scale, while 19 (73\%) trials [14, 16, 27, 28, 30, 32-36, 38, 40, 42, 44, 45, 47-49] had a relatively high methodological quality based on the modified Jadad scale. The randomization methods were adequate in 20 studies. Allocation concealment was adequate in 16 studies. Blinding was adequate in ten studies (Fig. 2). All studies had clear explanations for withdrawals and dropouts. Only six studies did not meet the ITT analysis criteria. The details of the quality assessment are shown in Table 2.

\section{Outcomes}

\section{Incidence of AKI}

Data regarding AKI incidence were available in all 26 studies, and the rate of AKI was significantly lower in the RIPC group than in the control group $[\mathrm{p}=0.01$; RR 0.79 (95\% CI 0.66-0.95), Fig. 3] (random model). However, it should be noted that different AKI definitions were applied in different studies. The AKI definitions used included the AKI Network (AKIN) criterion [16, 29, 38, 43, 47, 50], the Kidney Disease: Improving Global Outcomes (KDIGO) criterion [14, 41, 48], the RIFLE criterion [39, 46], postoperative serum creatinine $\geq 0.5 \mathrm{mg} / \mathrm{dl}$ or $\geq 25 \%$ above baseline [30, 34, 37, 40, 45], and others [27, 28, 31-33, 35, $36,42,44,49]$. We performed subgroup analyses based on the different AKI definitions. RIPC reduced AKI incidence as defined by a postoperative serum creatinine $\geq 0.5 \mathrm{mg} / \mathrm{dl}$ or $\geq 25 \%$ above baseline [p $=0.0002 ; \quad$ RR 0.42 $(0.27-0.67)$; heterogeneity $\chi^{2}=3.89, \mathrm{I}^{2}=0 \%, \mathrm{p}$ for heterogeneity $=0.42]$. However, RIPC did not reduce AKI incidence in the subgroups in terms of the other AKI definitions: AKIN criterion [ $p=0.56$; RR 0.87 (0.56-1.37); heterogeneity $\chi^{2}=12.22, \mathrm{I}^{2}=59 \%, \mathrm{p}$ for heterogeneity $=0.03], \quad$ KDIGO criterion $\quad[\mathrm{p}=0.32 ; \quad \mathrm{RR} \quad 0.83$ $(0.58-1.20)$; heterogeneity $\chi^{2}=6.76, \mathrm{I}^{2}=70 \%$, p for 


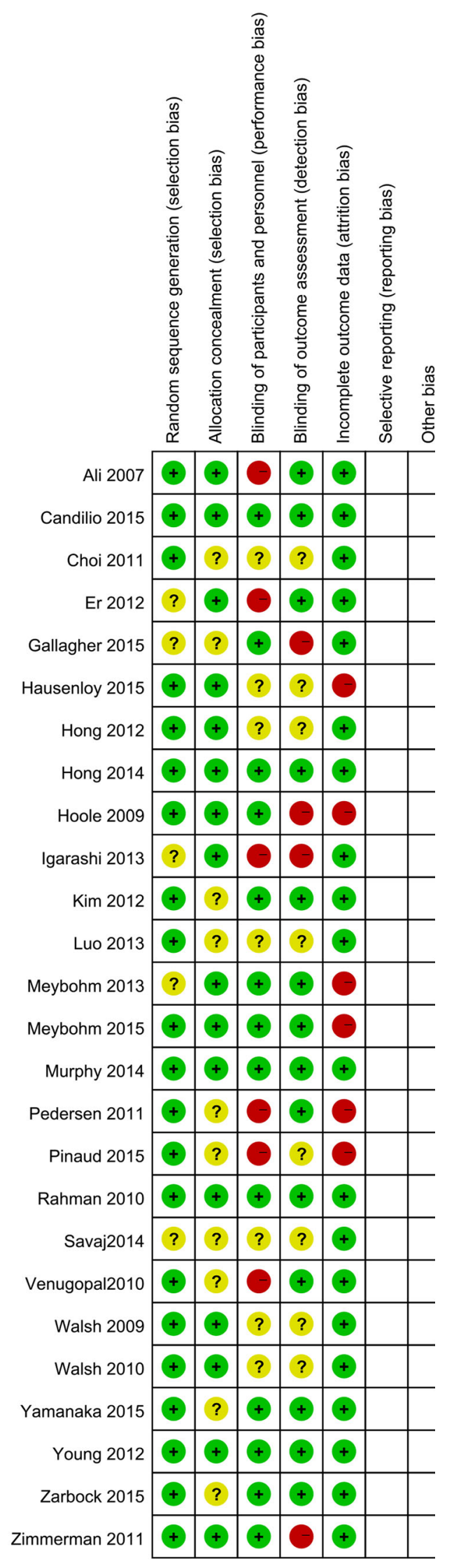

४Fig. 2 Risk of bias table: green low risk of bias, yellow unclear risk of bias, red high risk of bias (color figure online)

heterogeneity $=0.03]$, RIFLE criterion $[\mathrm{p}=0.37$; RR 0.87 (0.64-1.18); heterogeneity $\chi^{2}=0.06, \mathrm{I}^{2}=0 \%$, p for heterogeneity $=0.81]$, and others $[\mathrm{p}=0.34 ; \mathrm{RR} 0.83$ $(0.56-1.22)$; heterogeneity $\chi^{2}=18.85, \mathrm{I}^{2}=52 \%$, $\mathrm{p}$ for heterogeneity $=0.03$ ] (Fig. 4).

\section{In-hospital mortality}

In-hospital mortality was reported in 16 trials $[14,27,28$, $30,31,33,36,38-40,42-44,46,47,49]$, and there was no significant difference in mortality between the RIPC and control groups $[\mathrm{p}=0.97$; RR 1.01 (0.63-1.61); heterogeneity $\chi^{2}=11.84, \mathrm{I}^{2}=0 \%, \mathrm{p}$ for heterogeneity $=0.54$, Fig. 5].

\section{Change in renal biomarkers}

At 24 and $48 \mathrm{~h}$ after surgery, serum creatinine level was reported in five trials $[29,36,41,42,44]$ and five trials [29, $35,42,44,45]$, respectively. GFR was reported at 24 and $48 \mathrm{~h}$ after surgery in four trials [29, 35, 42, 44]. There were no significant differences between the two groups in serum creatinine (Scr) level or GFR at 24 or $48 \mathrm{~h}$ after surgery (Fig. 6).

\section{Initiation of renal replacement therapy}

Renal replacement therapy was reported in 15 trials [14, 27, 29-33, 38-40, 42, 44, 47, 50], and no significant difference was observed in the performance of renal replacement therapy between the RIPC group and the control group [p $=0.96$; RR $1.02(0.45-2.30)$; heterogeneity $\chi^{2}=21.35, \quad \mathrm{I}^{2}=58 \%, \quad \mathrm{p}$ for heterogeneity $=0.01$, Fig. 7].

\section{The lengths of hospital stay and ICU stay}

Four trials reported the length of hospital stay [29, 32, 36, 40], and three trials reported the length of ICU stay [29, 32, 36]. There was no significant difference in the length of hospital stay between the two groups $[\mathrm{p}=0.56$; mean difference 0.37 ( -0.87 to 1.61$)$; heterogeneity $\chi^{2}=5.64$, $\mathrm{I}^{2}=47 \%, \mathrm{p}$ for heterogeneity $\left.=0.13\right]$. The length of ICU stay was remarkably reduced in the RIPC group $[\mathrm{p}=0.008$; mean difference $-0.54(-0.95$ to -0.14$)$; heterogeneity $\chi^{2}=2.08, \mathrm{I}^{2}=4 \%, \mathrm{p}$ for heterogeneity $=0.35$, Fig. 8]; however, the number of trials was too small to observe a statistically significant difference. 
Table 2 Quality assessment of included trials

\begin{tabular}{|c|c|c|c|c|c|c|c|}
\hline References & Randomization method & $\begin{array}{l}\text { Allocation } \\
\text { concealment }\end{array}$ & Blinding & $\begin{array}{l}\text { Explanation for } \\
\text { withdrawals/dropouts }\end{array}$ & $\begin{array}{l}\text { Intention-to- } \\
\text { treat analysis }\end{array}$ & $\begin{array}{l}\text { Jadad } \\
\text { Score }\end{array}$ & $\begin{array}{l}\text { Modified } \\
\text { Jadad Score }\end{array}$ \\
\hline Ali et al. [27] & $\begin{array}{l}\text { Computer-generated random } \\
\text { list }\end{array}$ & $\begin{array}{l}\text { Sealed } \\
\text { envelopes }\end{array}$ & $\begin{array}{l}\text { Single } \\
\text { blind }\end{array}$ & Yes & Yes & 3 & 5 \\
\hline $\begin{array}{l}\text { Candilio } \\
\text { et al. [28] }\end{array}$ & $\begin{array}{l}\text { Computer-generated random } \\
\text { list }\end{array}$ & $\begin{array}{l}\text { Sealed } \\
\text { envelopes }\end{array}$ & $\begin{array}{c}\text { Double } \\
\text { blind }\end{array}$ & Yes & Yes & 5 & 7 \\
\hline $\begin{array}{l}\text { Choi et al. } \\
\text { [29] }\end{array}$ & $\begin{array}{l}\text { Computerized randomization } \\
\text { table }\end{array}$ & Unclear & Unclear & Yes & Yes & 3 & 3 \\
\hline Er et al. [30] & Unclear & $\begin{array}{l}\text { Sealed } \\
\text { envelopes }\end{array}$ & $\begin{array}{l}\text { Single } \\
\text { blind }\end{array}$ & Yes & Yes & 2 & 4 \\
\hline $\begin{array}{l}\text { Gallagher } \\
\text { et al. [31] }\end{array}$ & Unclear & Unclear & $\begin{array}{l}\text { Single } \\
\text { blind }\end{array}$ & Yes & Yes & 2 & 2 \\
\hline $\begin{array}{l}\text { Hausenloy } \\
\text { et al. [48] }\end{array}$ & By means of a secure website & $\begin{array}{l}\text { Sealed } \\
\text { envelopes }\end{array}$ & Unclear & Yes & No & 3 & 5 \\
\hline $\begin{array}{l}\text { Hong et al. } \\
\text { [32] }\end{array}$ & $\begin{array}{l}\text { Computer-generated random } \\
\text { list }\end{array}$ & $\begin{array}{l}\text { Sealed } \\
\text { envelopes }\end{array}$ & Unclear & Yes & Yes & 3 & 5 \\
\hline $\begin{array}{l}\text { Hong et al. } \\
\text { [33] }\end{array}$ & $\begin{array}{l}\text { Computer-generated random } \\
\text { list }\end{array}$ & $\begin{array}{l}\text { Sealed } \\
\text { envelopes }\end{array}$ & $\begin{array}{c}\text { Double } \\
\text { blind }\end{array}$ & Yes & Yes & 5 & 7 \\
\hline $\begin{array}{l}\text { Hoole et al. } \\
{[34]}\end{array}$ & $\begin{array}{l}\text { Computer-generated } \\
\text { randomization procedure }\end{array}$ & $\begin{array}{l}\text { Sealed } \\
\text { envelopes }\end{array}$ & $\begin{array}{l}\text { Single } \\
\text { blind }\end{array}$ & Yes & No & 3 & 5 \\
\hline $\begin{array}{l}\text { Igarashi et al. } \\
\text { [35] }\end{array}$ & Unclear & $\begin{array}{l}\text { Sealed } \\
\text { envelopes }\end{array}$ & $\begin{array}{l}\text { Non- } \\
\text { blind }\end{array}$ & Yes & Yes & 2 & 4 \\
\hline Kim et al. & $\begin{array}{l}\text { Computer-generated random } \\
\text { list }\end{array}$ & Unclear & $\begin{array}{c}\text { Double } \\
\text { blind }\end{array}$ & Yes & Yes & 5 & 5 \\
\hline $\begin{array}{l}\text { Luo et al. } \\
\text { [37] }\end{array}$ & $\begin{array}{l}\text { Simple digital method of } \\
\text { randomization }\end{array}$ & Unclear & Unclear & Yes & Yes & 3 & 3 \\
\hline $\begin{array}{l}\text { Meybohm } \\
\text { et al. [16] }\end{array}$ & Unclear & $\begin{array}{l}\text { Sealed } \\
\text { envelopes }\end{array}$ & $\begin{array}{r}\text { Double } \\
\text { blind }\end{array}$ & Yes & No & 4 & 6 \\
\hline $\begin{array}{l}\text { Meybohm } \\
\text { et al. [49] }\end{array}$ & $\begin{array}{l}\text { Performed by the Clinical Trial } \\
\text { Centre Leipzig }\end{array}$ & $\begin{array}{l}\text { Sealed } \\
\text { envelopes }\end{array}$ & $\begin{array}{c}\text { Double } \\
\text { blind }\end{array}$ & Yes & No & 5 & 7 \\
\hline $\begin{array}{l}\text { Murphy et al. } \\
{[38]}\end{array}$ & $\begin{array}{l}\text { Computer-generated random } \\
\text { list }\end{array}$ & $\begin{array}{l}\text { Sealed } \\
\text { envelopes }\end{array}$ & $\begin{array}{r}\text { Double } \\
\text { blind }\end{array}$ & Yes & Yes & 5 & 7 \\
\hline $\begin{array}{l}\text { Pedersen } \\
\text { et al. [39] }\end{array}$ & $\begin{array}{l}\text { Computerized randomization } \\
\text { table }\end{array}$ & Unclear & $\begin{array}{l}\text { Single } \\
\text { blind }\end{array}$ & Yes & No & 3 & 3 \\
\hline $\begin{array}{l}\text { Pinaud et al. } \\
\text { [50] }\end{array}$ & $\begin{array}{l}\text { Computerized randomization } \\
\text { table }\end{array}$ & Unclear & $\begin{array}{l}\text { Single } \\
\text { blind }\end{array}$ & Yes & No & 3 & 3 \\
\hline $\begin{array}{l}\text { Rahman et al. } \\
\text { [40] }\end{array}$ & $\begin{array}{l}\text { Computer-generated } \\
\text { randomization procedure }\end{array}$ & $\begin{array}{l}\text { Sealed } \\
\text { envelopes }\end{array}$ & $\begin{array}{r}\text { Double } \\
\text { blind }\end{array}$ & Yes & Yes & 5 & 7 \\
\hline $\begin{array}{l}\text { Savaj et al. } \\
\text { [41] }\end{array}$ & Unclear & Unclear & Unclear & Yes & Yes & 2 & 2 \\
\hline $\begin{array}{l}\text { Venugopal } \\
\text { et al. [43] }\end{array}$ & $\begin{array}{l}\text { Computer-generated random } \\
\text { list }\end{array}$ & Unclear & $\begin{array}{l}\text { Single } \\
\text { blind }\end{array}$ & Yes & Yes & 3 & 3 \\
\hline $\begin{array}{l}\text { Walsh et al. } \\
\text { [42] }\end{array}$ & $\begin{array}{l}\text { Computer-generated random } \\
\text { list }\end{array}$ & $\begin{array}{l}\text { Sealed } \\
\text { envelopes }\end{array}$ & Unclear & Yes & Yes & 3 & 5 \\
\hline $\begin{array}{l}\text { Walsh et al. } \\
\text { [44] }\end{array}$ & $\begin{array}{l}\text { Computer-generated random } \\
\text { list }\end{array}$ & $\begin{array}{l}\text { Sealed } \\
\text { envelopes }\end{array}$ & Unclear & Yes & Yes & 3 & 5 \\
\hline $\begin{array}{l}\text { Yamanaka } \\
\text { et al. [45] }\end{array}$ & $\begin{array}{l}\text { Computer-generated random } \\
\text { list }\end{array}$ & Unclear & $\begin{array}{r}\text { Double } \\
\text { blind }\end{array}$ & Yes & Yes & 5 & 5 \\
\hline $\begin{array}{l}\text { Young et al. } \\
{[46]}\end{array}$ & $\begin{array}{l}\text { Online randomization sequence } \\
\text { generator }\end{array}$ & $\begin{array}{l}\text { Sealed } \\
\text { envelopes }\end{array}$ & $\begin{array}{r}\text { Double } \\
\text { blind }\end{array}$ & Yes & Yes & 5 & 7 \\
\hline $\begin{array}{l}\text { Zarbock et al. } \\
\text { [14] }\end{array}$ & $\begin{array}{l}\text { Computer-generated random } \\
\text { list }\end{array}$ & Unclear & $\begin{array}{c}\text { Double } \\
\text { blind }\end{array}$ & Yes & Yes & 5 & 5 \\
\hline $\begin{array}{l}\text { Zimmerman } \\
\text { et al. [47] }\end{array}$ & $\begin{array}{l}\text { Block randomization generated } \\
\text { by study coordinator }\end{array}$ & $\begin{array}{l}\text { Sealed } \\
\text { envelopes }\end{array}$ & $\begin{array}{l}\text { Single } \\
\text { blind }\end{array}$ & Yes & Yes & 2 & 4 \\
\hline
\end{tabular}




\begin{tabular}{|c|c|c|c|c|c|c|c|c|c|c|}
\hline Study or Subgroup & $\begin{array}{r}\text { RIPC } \\
\text { Events }\end{array}$ & Total & $\begin{array}{l}\text { Contr } \\
\text { Events }\end{array}$ & $\begin{array}{l}\text { ol } \\
\text { Total }\end{array}$ & Weight & $\begin{array}{c}\text { Risk Ratio } \\
\text { M-H, Random, } 95 \% \mathrm{Cl}\end{array}$ & & $\begin{array}{r}\text { Risk } \\
\text { M-H. Rand }\end{array}$ & $\begin{array}{l}\text { Ratio } \\
\text { lom. } 95 \% \mathrm{Cl}\end{array}$ & \\
\hline Ali 2007 & 3 & 41 & 12 & 41 & $2.0 \%$ & $0.25[0.08,0.82]$ & & & & \\
\hline Candilio 2015 & 9 & 89 & 19 & 89 & $4.0 \%$ & $0.47[0.23,0.99]$ & & & & \\
\hline Choi 2011 & 14 & 38 & 12 & 38 & $4.8 \%$ & $1.17[0.62,2.18]$ & & & & \\
\hline Er 2012 & 6 & 50 & 20 & 50 & $3.4 \%$ & $0.30[0.13,0.68]$ & & & & \\
\hline Gallagher 2015 & 12 & 43 & 12 & 43 & $4.4 \%$ & $1.00[0.51,1.97]$ & & & & \\
\hline Hausenloy 2015 & 287 & 749 & 293 & 772 & $10.3 \%$ & $1.01[0.89,1.15]$ & & & & \\
\hline Hong 2012 & 1 & 35 & 4 & 35 & $0.7 \%$ & $0.25[0.03,2.13]$ & & & & \\
\hline Hong 2014 & 96 & 644 & 81 & 636 & $8.7 \%$ & $1.17[0.89,1.54]$ & & & $=$ & \\
\hline Hoole 2009 & 6 & 104 & 10 & 98 & $2.7 \%$ & $0.57[0.21,1.50]$ & & & & \\
\hline Igarashi 2013 & 2 & 30 & 8 & 30 & $1.4 \%$ & $0.25[0.06,1.08]$ & & & & \\
\hline Kim 2012 & 3 & 27 & 4 & 27 & $1.5 \%$ & $0.75[0.19,3.04]$ & & & & \\
\hline Luo 2013 & 2 & 101 & 1 & 104 & $0.6 \%$ & $2.06[0.19,22.36]$ & & & & \\
\hline Meybohm 2013 & 9 & 90 & 8 & 90 & $3.0 \%$ & $1.13[0.45,2.78]$ & & & & \\
\hline Meybohm 2015 & 42 & 692 & 35 & 693 & $6.7 \%$ & $1.20[0.78,1.86]$ & & & & \\
\hline Murphy 2014 & 16 & 31 & 11 & 31 & $5.2 \%$ & $1.45[0.81,2.61]$ & & & & \\
\hline Pedersen 2011 & 27 & 54 & 30 & 51 & $7.7 \%$ & $0.85[0.60,1.21]$ & & & & \\
\hline Pinaud 2015 & 13 & 50 & 12 & 49 & $4.4 \%$ & $1.06[0.54,2.09]$ & & & & \\
\hline Rahman 2010 & 5 & 75 & 8 & 77 & $2.3 \%$ & $0.64[0.22,1.87]$ & & & & \\
\hline Savaj2014 & 1 & 48 & 5 & 48 & $0.7 \%$ & $0.20[0.02,1.65]$ & & & & \\
\hline Venugopal2010 & 4 & 38 & 10 & 40 & $2.3 \%$ & $0.42[0.14,1.23]$ & & & & \\
\hline Walsh 2009 & 4 & 18 & 2 & 22 & $1.2 \%$ & $2.44[0.50,11.86]$ & & & & \\
\hline Walsh 2010 & 4 & 22 & 1 & 18 & $0.7 \%$ & $3.27[0.40,26.75]$ & & & & \\
\hline Yamanaka 2015 & 5 & 47 & 17 & 47 & $3.0 \%$ & $0.29[0.12,0.73]$ & & & & \\
\hline Young 2012 & 13 & 48 & 14 & 48 & $4.7 \%$ & $0.93[0.49,1.76]$ & & & & \\
\hline Zarbock 2015 & 45 & 120 & 63 & 120 & $8.6 \%$ & $0.71[0.54,0.95]$ & & & & \\
\hline Zimmerman 2011 & 12 & 59 & 28 & 59 & $5.3 \%$ & $0.43[0.24,0.76]$ & & & & \\
\hline Total $(95 \% \mathrm{CI})$ & & 3343 & & 3356 & $100.0 \%$ & $0.79[0.66,0.95]$ & & & & \\
\hline Total events & 641 & & 720 & & & & & & & \\
\hline \multicolumn{7}{|c|}{$\begin{array}{l}\text { Heterogeneity: } \mathrm{Tau}^{2}=0.08 ; \mathrm{Chi}^{2}=54.18, \mathrm{df}=25(\mathrm{P}=0.0006) ; \mathrm{I}^{2}=54 \% \\
\text { Test for overall effect: } \mathrm{Z}=2.45(P=0.01)\end{array}$} & 0.01 & $\begin{array}{l}0.1 \\
\text { Favours RIPC }\end{array}$ & $\begin{array}{lr}1 & 10 \\
& \text { Favours Cor }\end{array}$ & $\begin{array}{l}0 \\
\text { ontrol }\end{array}$ \\
\hline
\end{tabular}

Fig. 3 Meta-analysis of AKI incidence between RIPC and control groups. $C I$ confidence interval, $K H$ Knapp-Hartung method

\section{Subgroup analysis}

This meta-analysis of AKI incidence showed that RIPC reduces the perioperative incidence of AKI in cardiac and vascular surgery patients. However, there was high statistical heterogeneity among the included trials (heterogeneity $\chi^{2}=46.67, \mathrm{I}^{2}=53 \%, \quad \mathrm{p}$ for heterogeneity $=0.002$, Fig. 3). Since the different AKI definitions are based on different changes in serum creatinine from baseline, and contrast applications are specific clinical settings that could influence kidney function, we performed subgroup analyses of these two potential covariates. The results of the subgroup analysis were marginally significant (Figs. 4, 9); however, meta-regression analysis indicated that different AKI definitions were not the covariate contributing significantly to heterogeneity on the risk estimate for AKI incidence [coefficient $-0.39(-1.15$ to 0.38$) ; \mathrm{p}=0.56$ ]. We also did the meta-regression of contrast application conditions, and found that there was statistically significant difference in the risk estimate for AKI incidence [coefficient -0.22 ( -0.51 to 0.07$) ; \mathrm{p}=0.039$ ].

\section{Sensitivity analysis}

We used a fixed model and random model to analyze the different outcomes, and none of the results of the examined outcomes were different for these two models. These two models both indicated that remote ischemic preconditioning reduced the incidence of AKI in patients undergoing cardiovascular interventions.

\section{Discussion}

Many randomized controlled trials on remote ischemic preconditioning have been performed, and RIPC has been shown to ameliorate heart ischemia-reperfusion injury [51]. The commonly used RIPC methods are the placement of an inflatable tourniquet around the limbs and the crossclamping of the iliac arteries, both of which are noninvasive and nonpharmacological procedures. Cardiac and vascular surgery patients have a high risk of AKI [1], and AKI increases mortality [2]. However, currently, there are 


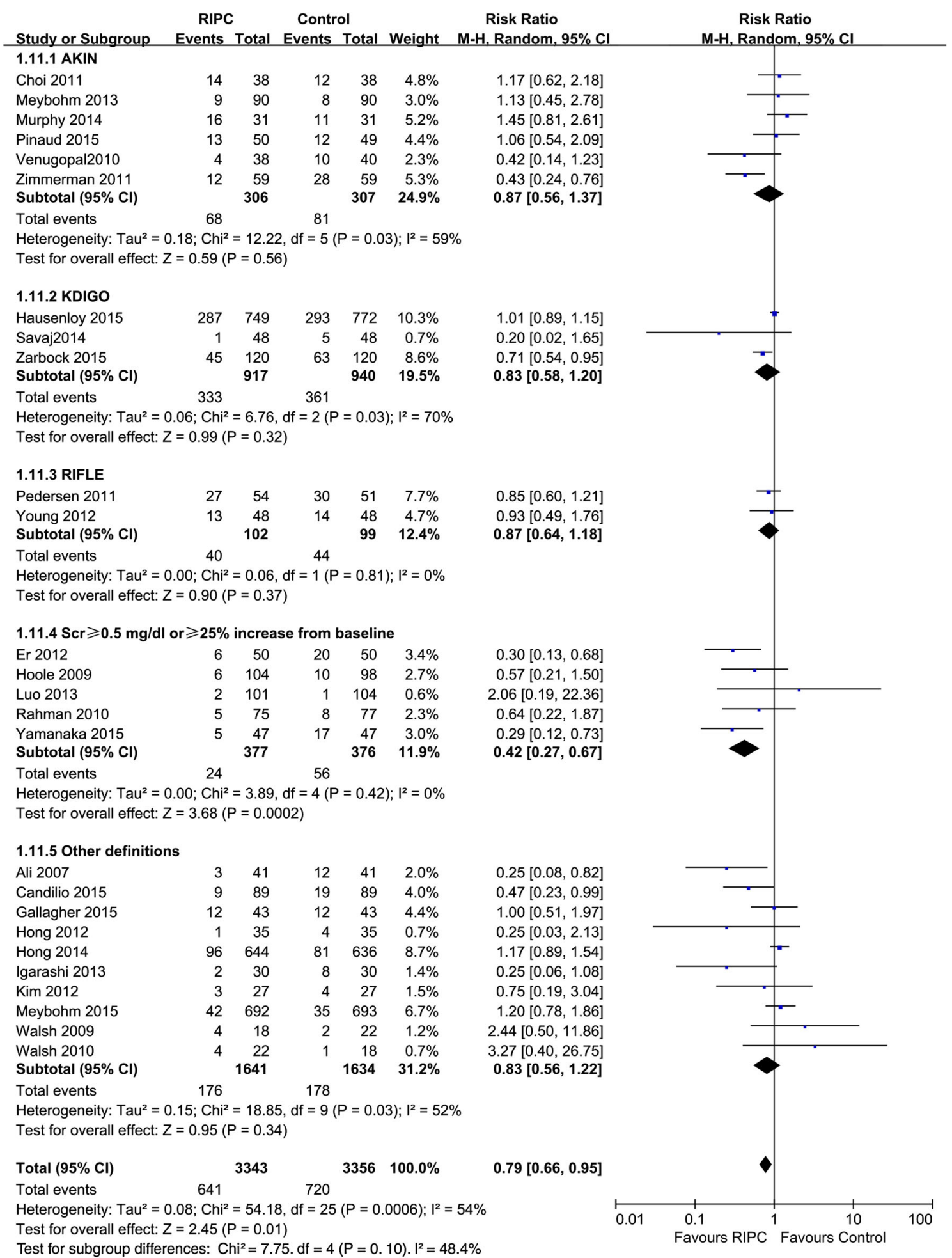

Fig. 4 Subgroup analysis for studies with different AKI definitions. $C I$ confidence interval, $K H$ Knapp-Hartung method

no effective clinical strategies for preventing the occurrence of AKI [5, 6, 12-14]. Remote ischemic preconditioning is a hot research area, and many researchers have applied this method to prevent AKI in cardiovascular surgery patients in recent years. However, disappointingly, the results of those studies do not clearly show whether RIPC 


\begin{tabular}{|c|c|c|c|c|c|c|c|c|c|c|}
\hline Study or Subgroup & $\begin{array}{l}\text { RIPC } \\
\text { Events }\end{array}$ & Total & $\begin{array}{l}\text { Contro } \\
\text { Events }\end{array}$ & $\begin{array}{l}\text { ol } \\
\text { Total }\end{array}$ & Weight & $\begin{array}{c}\text { Risk Ratio } \\
\text { M-H, Random, 95\% Cl }\end{array}$ & & $\begin{array}{r}\text { Risk } \\
\text { M-H, Rand }\end{array}$ & $\begin{array}{l}\text { Ratio } \\
\text { dom. } 95 \% \mathrm{Cl}\end{array}$ & \\
\hline Ali 2007 & 2 & 41 & 3 & 41 & $7.2 \%$ & $0.67[0.12,3.78]$ & & & & \\
\hline Candilio 2015 & 0 & 89 & 5 & 89 & $2.6 \%$ & $0.09[0.01,1.62]$ & & & & \\
\hline Er 2012 & 0 & 50 & 2 & 50 & $2.4 \%$ & $0.20[0.01,4.06]$ & & & & \\
\hline Gallagher 2015 & 2 & 43 & 2 & 43 & $6.0 \%$ & $1.00[0.15,6.78]$ & & & & \\
\hline Hong 2014 & 10 & 644 & 14 & 636 & $33.8 \%$ & $0.71[0.32,1.58]$ & & & & \\
\hline Kim 2012 & 0 & 27 & 0 & 27 & & Not estimable & & & & \\
\hline Meybohm 2015 & 9 & 692 & 4 & 693 & $15.9 \%$ & $2.25[0.70,7.28]$ & & & & \\
\hline Murphy 2014 & 3 & 31 & 1 & 31 & $4.5 \%$ & $3.00[0.33,27.29]$ & & & & \\
\hline Pedersen 2011 & 1 & 54 & 2 & 51 & $3.9 \%$ & $0.47[0.04,5.05]$ & & & & \\
\hline Rahman 2010 & 0 & 80 & 1 & 82 & $2.2 \%$ & $0.34[0.01,8.26]$ & & & & \\
\hline Venugopal2010 & 0 & 38 & 0 & 40 & & Not estimable & & & & \\
\hline Walsh 2009 & 1 & 18 & 0 & 22 & $2.2 \%$ & $3.63[0.16,84.11]$ & & & & \\
\hline Walsh 2010 & 3 & 22 & 0 & 18 & $2.6 \%$ & $5.78[0.32,105.12]$ & & & & \\
\hline Young 2012 & 1 & 48 & 1 & 48 & $2.9 \%$ & $1.00[0.06,15.53]$ & & & & \\
\hline Zarbock 2015 & 6 & 120 & 3 & 120 & $11.8 \%$ & $2.00[0.51,7.81]$ & & & & \\
\hline Zimmerman 2011 & 0 & 59 & 1 & 59 & $2.2 \%$ & $0.33[0.01,8.02]$ & & & & \\
\hline Total $(95 \% \mathrm{Cl})$ & & 2056 & & 2050 & $100.0 \%$ & $1.01[0.63,1.61]$ & & & & \\
\hline Total events & 38 & & 39 & & & & & & & \\
\hline $\begin{array}{l}\text { Heterogeneity: } \mathrm{Tau}^{2}= \\
\text { Test for overall effect: }\end{array}$ & $\begin{array}{l}0.00 ; \mathrm{Chi}^{2} \\
=0.04(\mathrm{~F}\end{array}$ & $\begin{array}{l}=11.84 \\
P=0.97\end{array}$ & $\begin{array}{l}\text { 4, } d f=13 \\
7)\end{array}$ & $(P=0$ & $.54) ; I^{2}=0$ & & 0.01 & $\begin{array}{l}0.1 \\
\text { Favours RIPC }\end{array}$ & $\begin{array}{l}1 \\
\text { Favours Co }\end{array}$ & $\begin{array}{l}10 \\
\text { ontrol }\end{array}$ \\
\hline
\end{tabular}

Fig. 5 Meta-analysis of in-hospital mortality between RIPC and control groups. $C I$ confidence interval, $K H$ Knapp-Hartung method

reduces AKI incidence in cardiac and vascular surgery patients. Meta-analyses performed by other teams also failed to reach a consistent conclusion. Yasin et al. [21] and Yang et al. [22] performed meta-analyses, and they both found no statistically significant differences in AKI incidence between cardiovascular surgery patients who did and did not undergo RIPC. However, a meta-analysis of 13 trials conducted by Yang et al. (1134 participants) showed that RIPC decreased the risk of AKI in cardiac and vascular surgery patients [23]. In addition, meta-analyses of other indices of renal impairment have not reached consistent conclusions [19, 21, 22, 52-54]. These inconsistent results may be due to the limitation of small sample size; therefore, larger samples and meta-analyses are needed.

This meta-analysis included 6699 participants in 26 trials who underwent cardiac or vascular interventions and were randomized to a RIPC group or control group. The results of our analysis reveal that RIPC significantly reduced AKI incidence in patients undergoing cardiac or vascular interventions $[p=0.01$; RR $0.79(0.66-0.95)]$. Because there was high statistical heterogeneity among the included trials, conclusions based on these results should be made with caution. The meta-analysis by Yang et al. indicated that the contrast medium intervention was not a covariate that significantly contributed to the heterogeneity in the risk estimate for AKI incidence, but the subgroup analysis of the contrast medium intervention in our study showed marginal statistical significance (Fig. 9). So we performed a meta-regression analysis, and the result showed that the contrast medium intervention was a covariate that significantly contributed to heterogeneity in the risk estimate for AKI incidence.

Although AKI incidence was reduced in the RIPC group, there were no significant differences in mortality or renal biomarkers between the two groups. Considering that not all of the included trials reported mortality or renal biomarkers, it is difficult to confirm whether RIPC has a kidney protective effect in patients undergoing cardiovascular interventions. Furthermore, other more sensitive indicators of early kidney damage, such as neutrophil gelatinase-associated lipocalin (NGAL) [55], Cys C [56] and urine output, were not available. Likewise, the use of renal replacement therapy and the length of hospital stay were not significantly different between the two groups. Length of ICU stay was shorter in the RIPC group, but that finding cannot be considered conclusive because only three trials reported valid data regarding ICU stay.

One trial only included patients without diabetes mellitus (DM) [43], whereas another trial only included patients with DM [41], and as DM is a potential risk factor for postoperative acute kidney injury in patients undergoing cardiac and vascular surgeries [57] the findings of our study may not be generalizable to non-diabetic patients. 


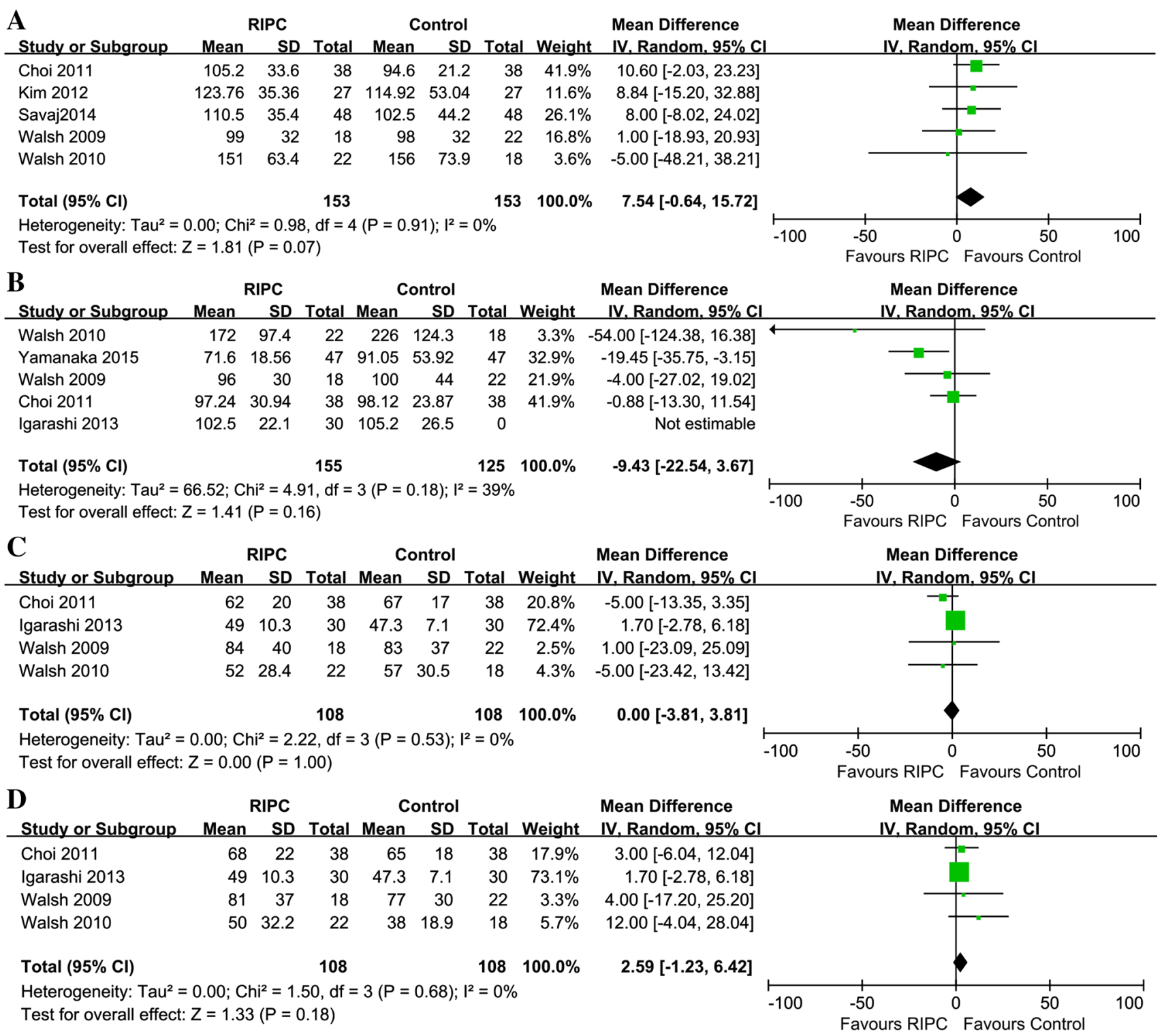

Fig. 6 Meta-analysis of mean differences in kidney biomarker levels between RIPC and control groups. a Serum creatinine (Scr) levels at $24 \mathrm{~h}$ postoperatively; b Scr levels at $48 \mathrm{~h}$ postoperatively;

Our meta-analysis has some limitations. First, surgery type, anesthesia and premedication varied between trials, which may have generated different risk levels of perioperative acute kidney injury incidence. Second, there was a high level of heterogeneity in the demographic data of the patients among the included trials: Also, the baseline serum creatinine level varied considerably between studies, which may indicate differences in basic renal function between studies. We should also note that the patients in the trial by Pedersen et al. [39] were children, while those in the other 25 studies were adults. Third, we did not limit this meta- c glomerular filtration rates (GFRs) at $24 \mathrm{~h}$ postoperatively; d GFRs at $48 \mathrm{~h}$ postoperatively. $C I$ confidence interval, $K H$ Knapp-Hartung method, $S D$ standard deviation

analysis to studies that examined one specific RIPC procedure: two of the included studies performed crossclamping of the iliac arteries [27, 44] instead of using an inflatable tourniquet around the limbs. Fourth, different AKI definitions were applied by the researchers of the different studies. All of these limitations may explain the high heterogeneity between studies besides the contrast application. Finally, only ten studies [14, 16, 28, 33, 36, 38, $40,45,46,49]$ were double-blind, and we think that the single-blind and non-blind studies may have influenced the results of this meta-analysis. 


\begin{tabular}{|c|c|c|c|c|c|c|c|c|c|c|}
\hline Study or Subgroup & $\begin{array}{r}\text { RIPC } \\
\text { Events }\end{array}$ & Total & $\begin{array}{l}\text { Contr } \\
\text { Events }\end{array}$ & $\begin{array}{l}\text { ol } \\
\text { Total }\end{array}$ & Weight & $\begin{array}{c}\text { Risk Ratio } \\
\mathrm{M}-\mathrm{H}, \text { Random, } 95 \% \mathrm{Cl}\end{array}$ & & $\begin{array}{r}\text { Risk } \\
\text { M-H, Randc } \\
\end{array}$ & $\begin{array}{l}\text { Ratio } \\
\text { lom. } 95 \% \mathrm{Cl}\end{array}$ & \\
\hline Ali 2007 & 0 & 41 & 0 & 41 & & Not estimable & & & & \\
\hline Choi 2011 & 0 & 38 & 0 & 38 & & Not estimable & & & & \\
\hline Er 2012 & 0 & 50 & 7 & 50 & $6.0 \%$ & $0.07[0.00,1.14]$ & & & & \\
\hline Gallagher 2015 & 2 & 43 & 0 & 43 & $5.5 \%$ & $5.00[0.25,101.18]$ & & & & \\
\hline Hong 2012 & 1 & 35 & 4 & 35 & $8.8 \%$ & $0.25[0.03,2.13]$ & & & & \\
\hline Hong 2014 & 26 & 644 & 15 & 636 & $19.9 \%$ & $1.71[0.92,3.20]$ & & & & \\
\hline Murphy 2014 & 7 & 31 & 0 & 31 & $6.1 \%$ & $15.00[0.89,251.77]$ & & & & \\
\hline Pedersen 2011 & 7 & 54 & 5 & 51 & $16.1 \%$ & $1.32[0.45,3.90]$ & & & & \\
\hline Pinaud 2015 & 0 & 50 & 2 & 49 & $5.5 \%$ & $0.20[0.01,3.98]$ & & & & \\
\hline Rahman 2010 & 3 & 80 & 1 & 82 & $8.3 \%$ & $3.08[0.33,28.95]$ & & & & \\
\hline Venugopal2010 & 0 & 38 & 0 & 40 & & Not estimable & & & & \\
\hline Walsh 2009 & 0 & 18 & 0 & 22 & & Not estimable & & & & \\
\hline Walsh 2010 & 2 & 22 & 0 & 18 & $5.6 \%$ & $4.13[0.21,80.91]$ & & & & \\
\hline Zarbock 2015 & 7 & 120 & 19 & 120 & $18.3 \%$ & $0.37[0.16,0.84]$ & & & & \\
\hline Zimmerman 2011 & 0 & 59 & 0 & 59 & & Not estimable & & & & \\
\hline Total $(95 \% \mathrm{CI})$ & & 1323 & & 1315 & $100.0 \%$ & $1.02[0.45,2.30]$ & & & & \\
\hline Total events & 55 & & 53 & & & & & & & \\
\hline \multicolumn{7}{|c|}{$\begin{array}{l}\text { Heterogeneity: } \mathrm{Tau}^{2}=0.76 ; \mathrm{Chi}^{2}=21.35, \mathrm{df}=9(P=0.01) ; \mathrm{I}^{2}=58 \% \\
\text { Test for overall effect: } Z=0.05(P=0.96)\end{array}$} & 0.01 & $\begin{array}{c}0.1 \\
\text { Favours RIPC }\end{array}$ & $\begin{array}{l}1 \\
\text { Favours Co }\end{array}$ & $\begin{array}{l}0 \\
\text { ontrol }\end{array}$ \\
\hline
\end{tabular}

Fig. 7 Meta-analysis of the use of renal replacement therapy between RIPC and control groups. $C I$ confidence interval, $K H$ Knapp-Hartung method

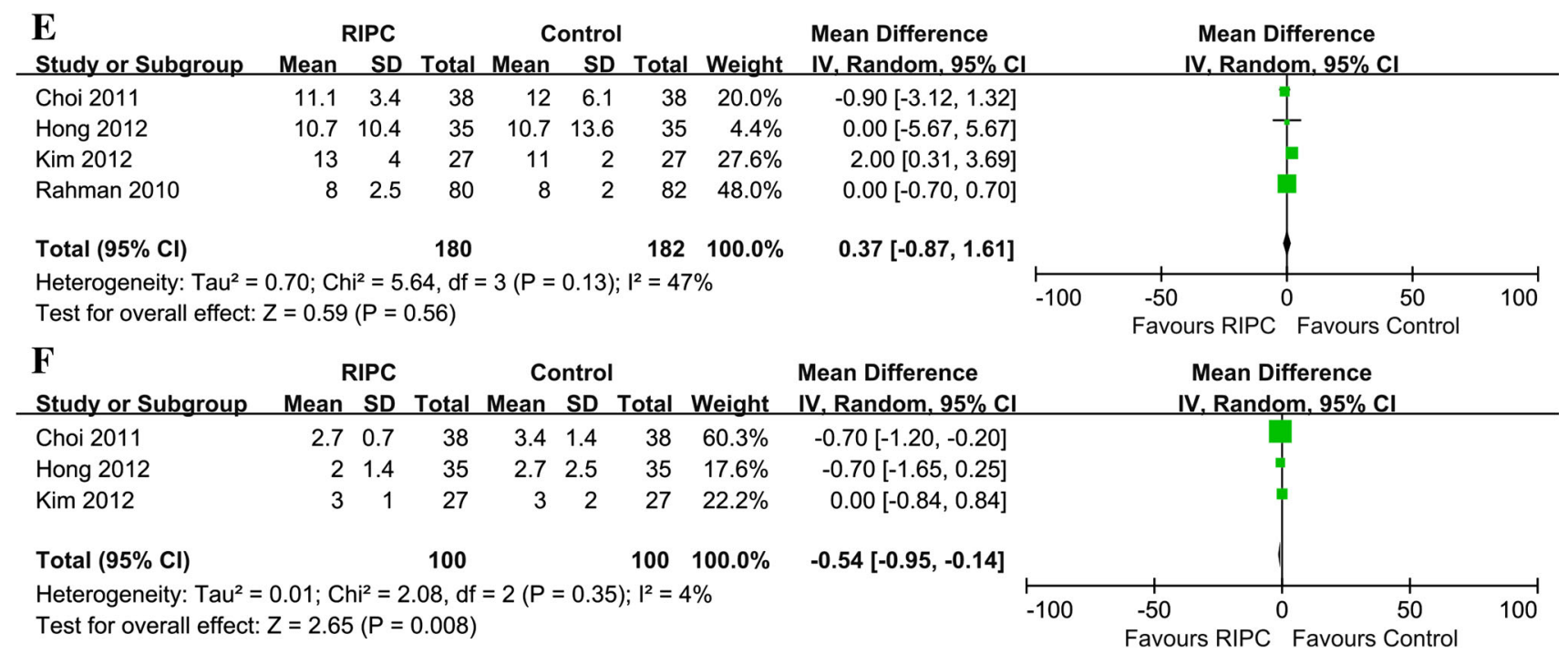

Fig. 8 Meta-analysis of e length of in-hospital stay and $\mathbf{f}$ length of intensive care unit (ICU) stay between RIPC and control groups. $C I$ confidence interval, $K H$ Knapp-Hartung method 


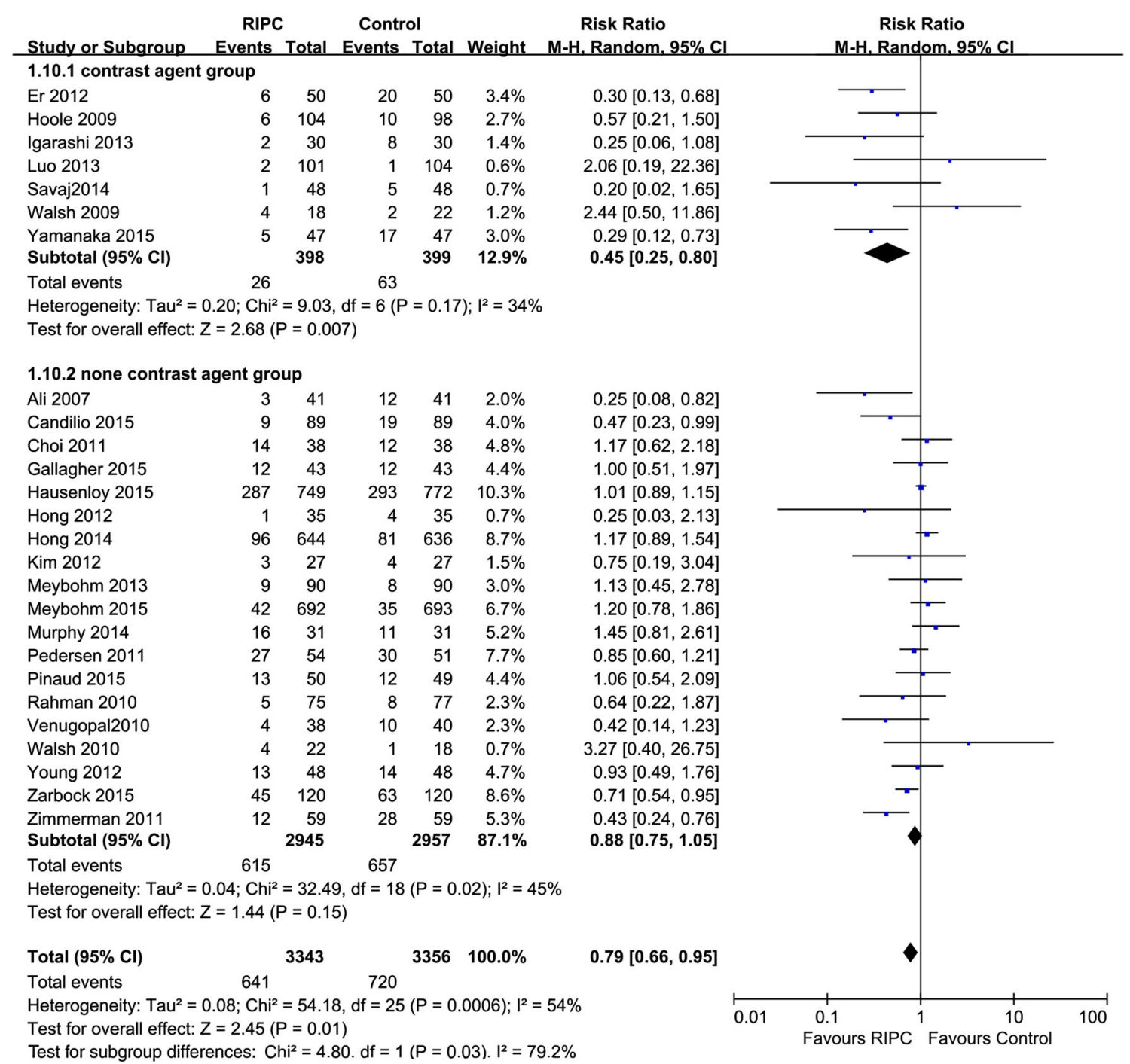

Fig. 9 Subgroup analysis based on the use of the contrast medium intervention. $C I$ confidence interval, $K H$ Knapp-Hartung method

\section{Conclusion}

Remote ischemic preconditioning can reduce the postoperative occurrence of acute kidney injury in cardiac and vascular surgery patients. However, considering the high heterogeneity among the 26 trials analyzed, we cannot draw a definitive conclusion regarding the value of RIPC at this time. A larger sample using a uniform AKI definition and RIPC method is needed to reach a more definitive conclusion.

\section{Compliance with ethical standards}

Funding This work was supported by Grants from the National Natural Science Foundation of China (81470938, 81200546, and 81300619).

Conflict of interest The authors declare that they have no conflict of interest.
Ethical standards All procedures performed in this study were in accordance with the ethical standards of the institutional and international research committee and with the 1964 Helsinki declaration and its later amendments or comparable ethical standards.

Research involving human participants and/or animals This article does not contain any studies with human participants or animals performed by any of the authors.

Informed consent Informed consent was obtained from all individual participants included in the study.

Open Access This article is distributed under the terms of the Creative Commons Attribution 4.0 International License (http://crea tivecommons.org/licenses/by/4.0/), which permits unrestricted use, distribution, and reproduction in any medium, provided you give appropriate credit to the original author(s) and the source, provide a link to the Creative Commons license, and indicate if changes were made. 


\section{References}

1. Thakar CV, Worley S, Arrigain S, Yared JP, Paganini EP (2007) Improved survival in acute kidney injury after cardiac surgery. Am J Kidney Dis 50(5):703-711. doi:10.1053/j.ajkd.2007.07.021

2. Conlon PJ, Stafford-Smith M, White WD, Newman MF, King S, Winn MP, Landolfo K (1999) Acute renal failure following cardiac surgery. Nephrol Dial Transplant 14:1158-1162

3. Tuttle KR, Worrall NK, Dahlstrom LR, Nandagopal R, Kausz AT, Davis CL (2003) Predictors of ARF after cardiac surgical procedures. Am J Kidney Dis 41:76-83. doi:10.1053/ajkd.2003. 50025

4. Bove T, Calabrò MG, Landoni G, Aletti G, Marino G, Crescenzi G, Rosica C, Zangrillo A (2004) The incidence and risk of acute renal failure after cardiac surgery. J Cardiothorac Vasc Anesth 18(4):442-445. doi:10.1053/j.jvca.2004.05.021

5. Rosner MH, Okusa MD (2006) Acute kidney injury associated with cardiac surgery. Clin J Am Soc Nephrol CJASN 1(1):19-32. doi:10.2215/CJN.00240605

6. Murray P (2008) Brain natriuretic peptide therapy to prevent acute kidney injury after cardiac surgery. Am J Kidney Dis 51(1):5-9. doi:10.1053/j.ajkd.2007.11.006

7. Loef BG, Epema AH, Navis G, Ebels T, Stegeman CA (2009) Postoperative renal dysfunction and preoperative left ventricular dysfunction predispose patients to increased long-term mortality after coronary artery bypass graft surgery. $\mathrm{Br} \mathrm{J}$ Anaesth 102(6):749-755. doi:10.1093/bja/aep088

8. Mariscalco G, Lorusso R, Dominici C, Renzulli A, Sala A (2011) Acute kidney injury: a relevant complication after cardiac surgery. Ann Thorac Surg 92(4):1539-1547. doi:10.1016/j.athor acsur.2011.04.123

9. Bansal S (2012) Post-surgical acute kidney injury. Clin Queries Nephrol 1(1):50-57. doi:10.1016/s2211-9477(11)70008-5

10. Huen SC, Parikh CR (2012) Predicting acute kidney injury after cardiac surgery: a systematic review. Ann Thorac Surg 93(1):337-347. doi:10.1016/j.athoracsur.2011.09.010

11. Ostermann ME, Taube D, Morgan CJ, Evans TW (2000) Acute renal failure following cardiopulmonary bypass: a changing picture. Intensive Care Med 26(5):565-571

12. Ranucci M, Soro G, Barzaghi N, Locatelli A, Giordano G, Vavassori A, Manzato A, Melchiorri C, Bove T, Juliano G, Uslenghi MF (2004) Fenoldopam prophylaxis of postoperative acute renal failure in high-risk cardiac surgery patients. Ann Thorac Surg 78(4):1332-1337. doi:10.1016/j.athoracsur.2004.02. 065 (discussion 1337-1338)

13. Haase M, Haase-Fielitz A, Plass M, Kuppe H, Hetzer R, Hannon C, Murray PT, Bailey MJ, Bellomo R, Bagshaw SM (2013) Prophylactic perioperative sodium bicarbonate to prevent acute kidney injury following open heart surgery: a multicenter doubleblinded randomized controlled trial. PLoS Med 10(4):e1001426. doi:10.1371/journal.pmed.1001426

14. Zarbock, Schmidt C, Van Aken H, Wempe C, Martens S, Zahn PK, Wolf B, Goebel U, Schwer CI, Rosenberger P (2015) Effect of remote ischemic preconditioning on kidney injury among highrisk patients undergoing cardiac surgery: a randomized clinical trial. JAMA 313:2133-2141. doi:10.1001/jama.2015.4189

15. Julier K, da Silva R, Garcia C, Bestmann L, Frascarolo P, Zollinger A, Chassot PG, Schmid ER, Turina MI, von Segesser LK, Pasch T, Spahn DR, Zaugg M (2003) Preconditioning by sevoflurane decreases biochemical markers for myocardial and renal dysfunction in coronary artery bypass graft surgery: a double-blinded, placebo-controlled, multicenter study. Anesthesiology 98:1315-1327

16. Meybohm P, Renner J, Broch O, Caliebe D, Albrecht M, Cremer J, Haake N, Scholz J, Zacharowski K, Bein B (2013)
Postoperative neurocognitive dysfunction in patients undergoing cardiac surgery after remote ischemic preconditioning: a doubleblind randomized controlled pilot study. PloS One 8:e64743. doi:10.1371/journal.pone.0064743

17. Kharbanda RK, Nielsen TT, Redington AN (2009) Translation of remote ischaemic preconditioning into clinical practice. Lancet 374:1557-1565

18. Gassanov N, Nia AM, Caglayan E, Er F (2014) Remote ischemic preconditioning and renoprotection: from myth to a novel therapeutic option? J Am Soc Nephrol JASN 25(2):216-224. doi:10. 1681/ASN.2013070708

19. Li L, Li G, Yu C, Li Y (2013) The role of remote ischemic preconditioning on postoperative kidney injury in patients undergoing cardiac and vascular interventions: a meta-analysis. J Cardiothor Surg 8:43

20. Hansen PR, Thibault H, Abdulla J (2010) Postconditioning during primary percutaneous coronary intervention: a review and meta-analysis. Int J Cardiol 144(1):22-25. doi:10.1016/j.ijcard. 2009.03.118

21. Haji Mohd Yasin NA, Herbison P, Saxena P, Praporski S, Konstantinov IE (2014) The role of remote ischemic preconditioning in organ protection after cardiac surgery: a meta-analysis. J Surg Res 186(1):207-216. doi:10.1016/j.jss.2013.09.006

22. Yang L, Wang G, Du Y, Ji B, Zheng Z (2014) Remote ischemic preconditioning reduces cardiac troponin I release in cardiac surgery: a meta-analysis. J Cardiothorac Vasc Anesth 28(3):682-689. doi:10.1053/j.jvca.2013.05.035

23. Yang Y, Lang XB, Zhang P, Lv R, Wang YF, Chen JH (2014) Remote ischemic preconditioning for prevention of acute kidney injury: a meta-analysis of randomized controlled trials. Am J Kidney Dis 64(4):574-583. doi:10.1053/j.ajkd.2014.04.029

24. Jadad AR, Moore RA, Carroll D, Crispin Jenkinson C, Reynolds DJM, Gavaghan DJ, McQuay HJ (1996) Assessing the quality of reports of randomized clinical trials: is blinding necessary? Control Clin Trials 17:1-12

25. Clark HD, Wells GA, Huët C, McAlister FA, Salmi LR, Fergusson D, Laupacis A (1999) Assessing the quality of randomized trials: reliability of the Jadad scale. Control Clin Trials 20:448-452

26. Oremus M, Wolfson C, Perrault A, Demers L, Momoli F, Moridea Y (2001) Interrater reliability of the modified Jadad quality scale for systematic reviews of Alzheimer's disease drug trials. Dement Geriatr Cogn Disord 12:232-236

27. Ali ZA, Callaghan CJ, Lim E, Ali AA, Nouraei SA, Akthar AM, Boyle JR, Varty K, Kharbanda RK, Dutka DP et al (2007) Remote ischemic preconditioning reduces myocardial and renal injury after elective abdominal aortic aneurysm repair: a randomized controlled trial. Circulation 116:I98-I105. doi:10.1161/ circulationaha.106.679167

28. Candilio L, Malik A, Ariti C, Barnard M, Di Salvo C, Lawrence D, Hayward M, Yap J, Roberts N, Sheikh A et al (2015) Effect of remote ischaemic preconditioning on clinical outcomes in patients undergoing cardiac bypass surgery: a randomised controlled clinical trial. Heart 101(3):185-192. doi:10.1136/heartjnl2014-306178

29. Choi YS, Shim JK, Kim JC, Kang KS, Seo YH, Ahn KR, Kwak YL (2011) Effect of remote ischemic preconditioning on renal dysfunction after complex valvular heart surgery: a randomized controlled trial. J Thorac Cardiovasc Surg 142:148-154. doi:10. 1016/j.jtcvs.2010.11.018

30. Er F, Nia AM, Dopp H, Dahlem KM, Caglayan E, Erdmann E, Gassanov N, Hellmich M, Burst V, Kubacki T et al (2012) Ischemic preconditioning for prevention of contrast medium-induced nephropathy: randomized pilot RenPro Trial (Renal Protection Trial). Circulation 126:296-303. doi:10.1161/ CIRCULATIONAHA.112.096370 
31. Gallagher SM, Jones DA, Kapur A, Wragg A, Harwood SM, Mathur R, Archbold RA, Uppal R, Yaqoob MM (2015) Remote ischemic preconditioning has a neutral effect on the incidence of kidney injury after coronary artery bypass graft surgery. Kidney Int 87:473-481. doi:10.1038/ki.2014.259

32. Hong DM, Jeon Y, Lee C-S, Kim HJ, Lee JM, Bahk JH, Kim KB, Hwang HY (2012) Effects of remote ischemic preconditioning with postconditioning in patients undergoing off-pump coronary artery bypass surgery. Circ J 76:884-890. doi:10.1253/circj.CJ11-1068

33. Hong DM, Lee EH, Kim HJ, Min JJ, Chin JH, Choi DK, Bahk JH, Sim JY, Choi IC, Jeon Y (2014) Does remote ischaemic preconditioning with postconditioning improve clinical outcomes of patients undergoing cardiac surgery? Remote ischaemic preconditioning with postconditioning outcome trial. Eur Heart $\mathbf{J}$ 35(3):176-183. doi:10.1093/eurheartj/eht346

34. Hoole SP, Heck PM, Sharples L, Khan SN, Duehmke R, Densem CG, Clarke SC, Shapiro LM, Schofield PM, O'Sullivan M (2009) Cardiac remote ischemic preconditioning in coronary stenting (CRISP Stent) study: a prospective, randomized control trial. Circulation 119:820-827. doi:10.1161/CIRCULATIONAHA. 108.809723

35. Igarashi G, Iino K, Watanabe H, Ito H (2013) Remote ischemic pre-conditioning alleviates contrastinduced acute kidney injury in patients with moderate chronic kidney disease. Circ J 77:3037-3044. doi:10.1253/circj.CJ-13-0171

36. Kim JC, Shim JK, Lee S, Yoo YC, Yang SY, Kwak YL (2012) Effect of combined remote ischemic preconditioning and postconditioning on pulmonary function in valvular heart surgery. Chest 142(2):467-475. doi:10.1378/chest.11-2246

37. Luo SJ, Zhou YJ, Shi DM, Ge HL, Wang JL, Liu RF (2013) Remote ischemic preconditioning reduces myocardial injury in patients undergoing coronary stent implantation. Can J Cardiol 29(9):1084-1089. doi:10.1016/j.cjca.2012.11.022

38. Murphy N, Vijayan A, Frohlich S, O'Farrell F, Barry M, Sheehan S, Boylan J, Conlon N (2014) Remote ischemic preconditioning does not affect the incidence of acute kidney injury after elective abdominal aortic aneurysm repair. J Cardiothorac Vasc Anesth 28:1285-1292. doi:10.1053/j.jvca.2014.04.018

39. Pedersen (2011) Failure of remote ischemic preconditioning to reduce the risk of postoperative acute kidney injury in children undergoing operation for complex congenital heart disease: a randomized single-center study. J Thorac Cardiovasc Surg. doi:10.1016/j.jtcvs.2011.08.044

40. Rahman IA, Mascaro JG, Steeds RP, Frenneaux MP, Nightingale P, Gosling P, Townsend P, Townend JN, Green D, Bonser RS (2010) Remote ischemic preconditioning in human coronary artery bypass surgery: from promise to disappointment? Circulation 122:S53-S59. doi:10.1161/CIRCULATIONAHA.109. 926667

41. Savaj S, Savoj J, Jebraili I, Sezavar SH (2014) Remote ischemic preconditioning for prevention of contrast-induced acute kidney injury in diabetic patients. Iran J Kidney Dis 8:457-460

42. Walsh SR, Jonathan R, Tang TY, Sadat U, David G, Lapsley M, Norden AG, Varty K, Hayes PD, Gaunt ME (2009) Remote ischemic preconditioning for renal and cardiac protection during endovascular aneurysm repair: a randomized controlled trial. J Endovasc Ther 16:680-689

43. Venugopal V, Laing CM, Ludman A, Yellon DM, Hausenloy D (2010) Effect of remote ischemic preconditioning on acute kidney injury in nondiabetic patients undergoing coronary artery bypass graft surgery: a secondary analysis of two small randomized trials. Am J Kidney Dis 56:1043-1049. doi:10.1053/j. ajkd.2010.07.014

44. Walsh SR, Sadat U, Boyle JR, Tang TY, Lapsley M, Norden AG, Gaunt ME (2010) Remote ischemic preconditioning for renal protection during elective open infrarenal abdominal aortic aneurysm repair: randomized controlled trial. Vasc Endovasc Surg 44(5):334-340. doi:10.1177/15385744410370788

45. Yamanaka T, Kawai Y, Miyoshi T, Mima T, Takagaki K, Tsukuda S, Kazatani Y, Nakamura K, Ito H (2015) Remote ischemic preconditioning reduces contrast-induced acute kidney injury in patients with ST-elevation myocardial infarction: a randomized controlled trial. Int J Cardiol 178:136-141. doi:10.1016/j.ijcard. 2014.10.135

46. Young PJ, Garden A, Horrocks C, La Flamme A, Mahon B, Miller J, Pilcher J, Weatherall M, Williams J, Young W, Beasley R (2012) A pilot study investigating the effects of remote ischemic preconditioning in high-risk cardiac surgery using a randomised controlled double-blind protocol. Basic Res Cardiol 107(3):256. doi:10.1007/s00395-012-0258-4

47. Zimmerman RF, Ezeanuna PU, Kane JC, Cleland CD, Kempananjappa TJ, Lucas FL, Kramer RS (2011) Ischemic preconditioning at a remote site prevents acute kidney injury in patients following cardiac surgery. Kidney Int 80:861-867. doi:10.1038/ ki.2011.156

48. Hausenloy DJ, Candilio L, Evans R, Ariti C, Jenkins DP, Kolvekar S, Knight R, Kunst G, Laing C, Nicholas J, Pepper J, Robertson S, Xenou M, Clayton T, Yellon DM, ET Investigators (2015) Remote ischemic preconditioning and outcomes of cardiac surgery. N Engl J Med 373(15):1408-1417. doi:10.1056/ NEJMoa1413534

49. Meybohm P, Bein B, Brosteanu O, Cremer J, Gruenewald M, Stoppe C, Coburn M, Schaelte G, Boning A, Niemann B et al (2015) A multicenter trial of remote ischemic preconditioning for heart surgery. N Engl J Med 373(15):1397-1407. doi:10.1056/ NEJMoa1413579

50. Pinaud F, Corbeau JJ, Baufreton C, Binuani JP, De Brux JL, Fouquet O, Angoulvant D, Furber A, Prunier F (2016) Remote ischemic preconditioning in aortic valve surgery: results of a randomized controlled study. J Cardiol 67(1):36-41. doi:10.1016/ j.jjcc.2015.06.007

51. Marczak J, Nowicki R, Kulbacka J, Saczko J (2012) Is remote ischaemic preconditioning of benefit to patients undergoing cardiac surgery? Interact Cardiovasc Thorac Surg 14(5):634-639. doi:10.1093/icvts/ivr123

52. Brevoord D, Kranke P, Kuijpers M, Weber N, Hollmann M, Preckel B (2012) Remote ischemic conditioning to protect against ischemia-reperfusion injury: a systematic review and meta-analysis. PLoS One 7(7):e42179. doi:10.1371/journal.pone. 0042179

53. D'Ascenzo F, Cavallero E, Moretti C, Omede P, Sciuto F, Rahman IA, Bonser RS, Yunseok J, Wagner R, Freiberger $\mathrm{T}$ et al (2012) Remote ischaemic preconditioning in coronary artery bypass surgery: a meta-analysis. Heart 98(17):1267-1271. doi:10. 1136/heartjnl-2011-301551

54. Alreja G, Bugano D, Lotfi A (2012) Effect of remote ischemic preconditioning on myocardial and renal injury: meta-analysis of randomized controlled trials. J Invasive Cardiol 24(2):42-48

55. Koyner JL, Garg AX, Coca SG, Sint K, Thiessen-Philbrook H, Patel UD, Shlipak MG, Parikh CR, Consortium T-A (2012) Biomarkers predict progression of acute kidney injury after cardiac surgery. J Am Soc Nephrol JASN 23(5):905-914. doi:10. 1681/ASN.2011090907

56. Rule AD, Lieske JC (2011) Cystatin C is more than GFR, and this may be a good thing. J Am Soc Nephrol JASN 22(5):795-797. doi:10.1681/ASN.2011030288

57. Hong S, Youn Y-N, Yoo K-J (2010) Metabolic syndrome as a risk factor for postoperative kidney injury after off-pump coronary artery bypass surgery. Circ J 74(6):1121-1126. doi:10.1253/ circj.CJ-09-0842 